\title{
Antiproliferative Properties of Vinyl Dipeptides: Synthesis and MCF-7 Cell Line Testing
}

\author{
Ashraf Hassan Bayoumi \\ Department of Organic Chemistry, Faculty of Pharmacy, Al-Azhar University, Cairo, Egypt \\ Email: Bayoumi.ashraf@hotmail.com
}

Received October 18, 2012; revised November 23, 2012; accepted December 2, 2012

\begin{abstract}
Peptide mimics derived with close structure to peptide have vast utility because they are expected to interfere with biological targets while having superior drug-like properties if compared to peptides. In this work, novel vinyl dipeptides which are different in a double bond between the $\alpha$-carbon of peptide and $\mathrm{C} 1$ of its side chain. Added to that, suitable substituents were selected to harness drug-like properties. The compounds were found to have moderate activities when tested against MCF-7 breast cancer cell line. For instance, the adamantyl analogue 2-(benzoylamino)-3-(2-furyl)- $N$ (1-adamantyl) propenamide (1c) and the heterocyclic analogue 2-(Benzoylamino)-3-(2-furyl)- $N$-[2-(5-cyanothia-zol-2yl)] propenamide (10) exhibited inhibition potency at 27.4 and $37.8 \mu \mathrm{M}$, respectively.
\end{abstract}

Keywords: Piptidomimetics; Vinyl Dipeptides; 2-Aminopropenamide; Antiproliferative; Breast Cancer; Furan Derivatives

\section{Introduction}

Peptides are involved in most of uncontrolled cell division activities that lead to several malignant and immunologic diseases [1]. Enzymes in rapidly dividing cells utilize peptides as substrates or sometimes as activating agents. One of the established strategies to combat proliferative diseases is to interfere with peptides in their binding with active sites using mimic molecules leading to interruption of cell division activities [2]. The vinylpeptide 2-acylamino- $N$-substituted acrylamide scaffold 1 (Figure 1) has proven activities in several disor-

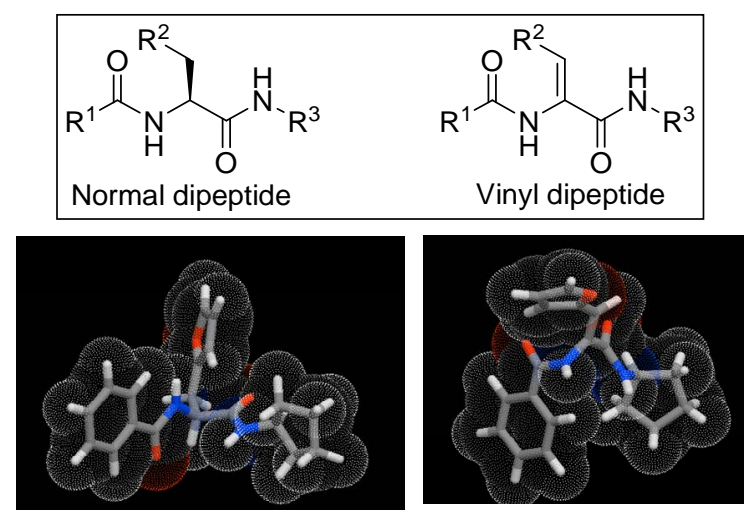

Figure 1. Peptidomimetic vinyl dipeptides. Top left normal dipeptide (left) and three dimensional of a representative example while the vinyl peptide is shown to the right. The three dimensional structure was generated using 3D-Galaxy module of Molinispiration. ders such as malaria [3], respiratory disease [4], inflammation [5], viral diseases [6]. Compounds belonging to this pseudopeptide scaffold were synthesized and tested for their antiproliferative activities in different cancer cell lines $[7,8]$. Few reports investigated geranylgeranyltransferase [5], dishevelled PDZ domain [9] as possible targets for tumor inhibition mechanisms of vinyl peptides.

We envisaged that the versatility of vinyl peptides enables them to have polypharmacology profile [10]. Therefore, preliminary screening of vinyl dipeptides should start with cell line inhibition tests rather than screening against specific protein target(s). In addition, the vinyl dipeptides showed ability to penetrate cells in several reports and in some cases they had in vivo potencies efficacies [11,12].

We believe that this scaffold is indeed interesting for development of drug-like viable leads with potent antiproliferative activity and therefore, we decided to investigate some of its derivatives against breast cancer cell lines.

\section{Results and Discussion}

\subsection{Chemistry}

Compounds were synthesized as described in Scheme 1 starting from in-house prepared hippuric acid 2 which was reacted with 2-furaldehyde to afford 4-furfurylidene-2-phenyloxazol-5(4H)-one (3) under standard Er- 
lenmeyer conditions [13]. The lactone intermediate $\mathbf{3}$ was heated with a set of different aliphatic, aromatic and heterocyclic amines under microwave conditions in $\mathrm{N}, \mathrm{N}$ dimethylformamide (DMF) to give the vinylamide titled compounds except the 4-aminophenol that underwent dehydration and ring closure, under the same experimental conditions, giving the $5(4 H)$-imidazolone 4 . The ${ }^{1} \mathrm{H}$ NMR, HPLC/ES-MS and elemental analyses supported this interpretation. The imidazolone $\mathbf{4}$ did not show the two distinct amide $\mathrm{NH}$ signals characteristic for the vinyl diamide open analogues. In addition, ES-MS showed molecular ion peak at $m / z(\mathrm{M}-18)^{+}$off the expected open structure. FT-IR of open analogues showed characteristic $\mathrm{NH}$ bands in the range of $3300-3100 \mathrm{~cm}^{-1}$. The ringclosure observed in case of compound $\mathbf{4}$ was referred to the strong positive resonance effect of the parahydroxy group that increases the nucleophilicity of the $\mathrm{NH}$ moiety facilitating the cyclization process as depicted in Scheme 2.

\subsection{MCF-7 Cell Line Test}

Breast cancer is our focus in this work because it is the most frequently diagnosed cancer and the leading cause of cancer death in females worldwide. About half of the breast cancer cases and $60 \%$ of the deaths are estimated to occur in economically developing countries [14].

Thus, inhibition of breast cancer cell line (MCF-7) was determined using SRB method as previously described by Skehan et al. [15]. Compounds 1c, 1d, 1k, 10 exhibited promising inhibition potency $(<50 \mu \mathrm{M})$ as described in Tables 1(a) and (b).
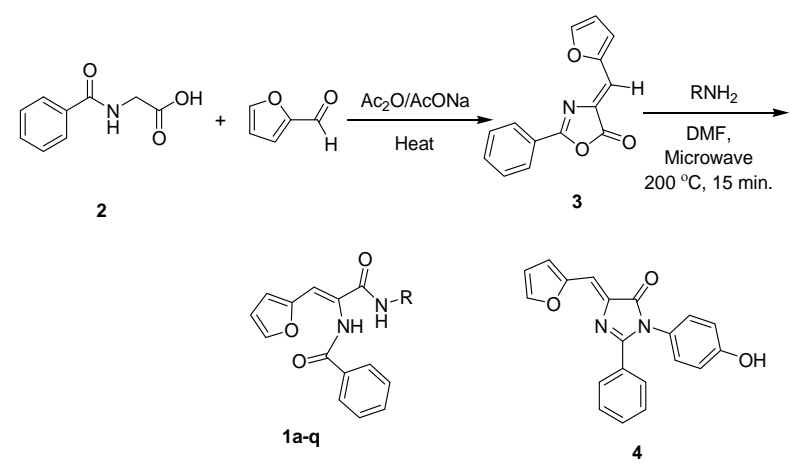

Scheme 1. Synthesis of vinyl diamide compounds.

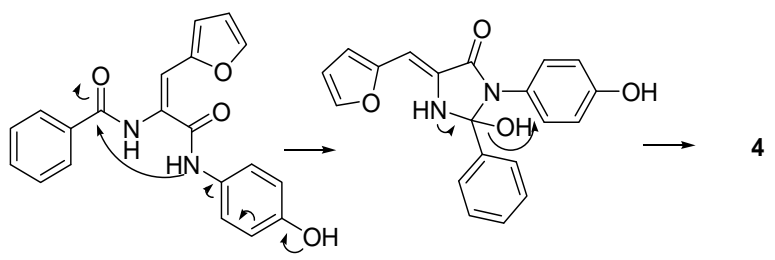

Scheme 2. Proposed mechanism of formation of compound 4.
Table 1. (a), (b) Molecular properties and MCF-7 breast cancer cell line inhibition testing of title compounds.

(a)

\begin{tabular}{|c|c|c|c|c|}
\hline Cpd & $\mathbf{R}$ & M.Wt. & $\begin{array}{c}\text { MCF7 } \\
\text { IC }_{50} \boldsymbol{\mu M} \\
\end{array}$ & $\operatorname{miLog} P^{1}$ \\
\hline 1a & Et & 284.3 & 509.3 & 2.150 \\
\hline $1 b$ & Cyclopentyl & 324.2 & 241.8 & 3.170 \\
\hline 1c & Adamantyl & 390.5 & 27.42 & 4.698 \\
\hline 1d & $\mathrm{Ph}$ & 332.4 & 47.03 & 3.471 \\
\hline 1e & 3-MePh & 346.4 & NA & 3.896 \\
\hline 1f & 4-MePh & 346.4 & 129.4 & 3.920 \\
\hline $1 \mathrm{~g}$ & 3-FPh & 350.3 & 74.14 & 3.611 \\
\hline 1h & 4-FPh & 350.3 & 940.4 & 3.635 \\
\hline $\mathbf{1 i}$ & 3-ClPh & 366.6 & 139.4 & 4.125 \\
\hline $1 j$ & 3-MeOPh & 362.4 & 82.13 & 3.504 \\
\hline $1 \mathrm{k}$ & $3-\mathrm{OH}$ & 348.1 & 47.37 & 2.968 \\
\hline 11 & 4- $\mathrm{NH}_{2} \mathrm{SO}_{2} \mathrm{Ph}$ & 411.4 & 89.59 & 2.165 \\
\hline $1 \mathrm{~m}$ & $4-\mathrm{NO}_{2} \mathrm{Ph}$ & 377.4 & 56.48 & 3.430 \\
\hline 1n & 4-FBn & 364.4 & 695.3 & 3.336 \\
\hline 10 & 5-CN-2-thiazolyl & 364.4 & 37.88 & 2.520 \\
\hline $1 p$ & 2-Pyridyl & 333.1 & 697.3 & 3.332 \\
\hline $1 q$ & 3-Pyridyl & 333.1 & 741.7 & 2.949 \\
\hline \multirow[t]{2}{*}{4} & 4-OHPh & 330.3 & $>1000$ & 3.330 \\
\hline & Doxorubicin & & 1.172 & \\
\hline
\end{tabular}

(b)

\begin{tabular}{ccccccc}
\hline Cpd & TPSA $^{2}$ & $\begin{array}{c}\text { H-Bond } \\
\text { Acceptor }\end{array}$ & $\begin{array}{c}\text { H-Bond } \\
\text { Donor }\end{array}$ & RBs $^{3}$ & $\begin{array}{c}\text { Ro5 } \\
\text { Violations }\end{array}$ & $\begin{array}{c}\text { Chimney } \\
\text { Test }\end{array}$ \\
\hline 1a & 71.33 & 5 & 2 & 5 & 0 & $5 / 6$ \\
1b & 71.33 & 5 & 2 & 5 & 0 & $6 / 6$ \\
1c & 71.33 & 5 & 2 & 5 & 0 & $6 / 6$ \\
1d & 71.33 & 5 & 2 & 5 & 0 & $4 / 6$ \\
1e & 71.33 & 5 & 2 & 5 & 0 & $6 / 6$ \\
1f & 71.33 & 5 & 2 & 5 & 0 & $5 / 6$ \\
1g & 71.33 & 5 & 2 & 5 & 0 & $4 / 6$ \\
1h & 71.33 & 5 & 2 & 5 & 0 & $6 / 6$ \\
1i & 71.33 & 5 & 2 & 5 & 0 & $6 / 6$ \\
1j & 80.57 & 6 & 2 & 6 & 0 & $5 / 6$ \\
1k & 91.56 & 6 & 3 & 5 & 0 & $6 / 6$ \\
1l & 131.50 & 8 & 4 & 6 & 0 & $3 / 6$ \\
1m & 117.16 & 8 & 2 & 6 & 0 & $5 / 6$ \\
1n & 71.33 & 5 & 2 & 6 & 0 & $\mathrm{~N} / \mathrm{A}$ \\
$\mathbf{1 0}$ & 108.02 & 7 & 2 & 5 & 0 & $5 / 6$ \\
1p & 60.92 & 5 & 0 & 3 & 0 & $4 / 6$ \\
1q & 60.92 & 5 & 0 & 3 & 0 & $3 / 6$ \\
$\mathbf{4}$ & 68.27 & 5 & 1 & 3 & 0 & $6 / 6$ \\
\hline
\end{tabular}

${ }^{1}$ miLogP: Method for $\log \mathrm{P}$ prediction developed at Molinispiration (miLogP2.2 November 2005); ${ }^{2}$ TPSA: Topological Polar Surface Area [16]; ${ }^{3} \mathrm{RBs}$ : Number of Rotatable Bonds. 


\subsection{Discussion}

The goal of this work is to explore the antiproliferative activity and to find some lead structures as a starting point for future disciplined search of optimized drug candidates. Cytotoxic evaluation of the prepared vinyl dipeptides using the MCF-7 cell line test revealed moderate cellular inhibition potencies of vinyl dipeptide compounds as measured by their $\mathrm{IC}_{50}$ values (Table 1). For $\mathrm{N}$-aliphatic series, it is clear that increasing the bulkiness of the alicyclic group led to a significant increase in the anticancer potency. The adamantyl derivative 1c had approximately 20 -fold potency of $N$-ethyl derivative 1 a while it was observed to have 10 times better cytotoxicity than the $N$-cyclopentyl analogue $1 \mathrm{~b}$. The $N$-benzenoid series showed inconsistent results indicating that the aryl group may not have a role in binding with the receptor. Another possibility of the inconsistent SAR observations is multiple modes of actions and interfering with more than one biological target. This is agreeing with our polypharmacology hypothesis for these compounds [10]. The three dimensional illustration of normal dipeptide vs. vinyl dipeptide of compound $1 \mathrm{~b}$ (Figure 1) gave insight to differences between the two structures and hence, their biological actions. It is noteworthy to underscore the rigidity added by this double bond in our pseudopeptide scaffold.

Concerning possible toxicities, we performed Chimney Test to perform preliminary evaluation of neuromotor acute toxicity [17]. The most potent anti-proliferative compound 1c passed the test as all animals climbed the tube successfully within 30 seconds. The second potent compound 1o showed weak neurotoxicity evidenced by results of Chimney Test as five out of six animals passed the test. The ring-closed analogue 4 showed only weak antitumor activity compared to vinyl dipeptides 1 but it is not an indication of activity trend of this different chemical species. Initial results above encouraged us to decide the follow-up studies on the open-chain series 1 .

Towards developing drug-like molecules, we subjected all synthesized compounds to a variety of molecular descriptors calculations (Table 1). No violation for Lipinski's Rule of 5 (Ro5) was observed in any compound [18]. Another tactic in this present study is to fix two diversity points featured in the scaffold 1 (Figure 1) by using benzoyl and furfurylidene groups at positions $\mathrm{R}^{1}$ and $\mathrm{R}^{2}$. Therefore, our focus was to define SAR by different amines on one diversity point. Our selection of the hydrophilic furyl group was based on keeping control on physico-chemical properties by balancing the hydrophobic $N$-benzoyl substituent. This balance allowed using wide range of hydrophobic amines such as the adamantyl derivative 1c as well as hydrophilic arylamines such as the cyanothiazolyl analogue 1o on the $N$-terminal of the scaffold without violating the principles of druglikeness [19].

\section{Conclusion}

The newly prepared pseudopeptides have shown promising activities as anticancer agents. It is noteworthy to mention that all significantly active compounds $(<50 \mu \mathrm{M})$ are having molecular weight under 400 mass unit. This is important because future research to discover more potent leads may require adding further molecular mass without concern of breaching the Ro5. Plans for future work are clear: Studying SAR on $\mathrm{R}^{1}$ and $\mathrm{R}^{2}$ that cover broader molecular space. Driving potency to comparable level to doxorubicin should be reached and then followed by determining the anticancer mechanism by deeper biochemical and genetic studies.

\section{Experimental}

All melting points were uncorrected and measured using capillary melting point instrument (Stuart, UK). IR spectra were recorded as potassium bromide pellets on a Perkin-Elmer 1650 spectrophotometer (USA), Faculty of Science, Cairo University, Cairo, Egypt. ${ }^{1} \mathrm{H}-\mathrm{NMR}$ spectra were determined on Avance III $600 \mathrm{MHz}$ spectrometer (Bruker, Germany) and chemical shifts were expressed as ppm against TMS as internal reference (King Fahd Center for Medical Research, King Abdulaziz University, Jeddah, Saudi Arabia). Analyses by TSQ Quantum Access MAX Triple Stage Quadrupole Mass Spectrometer, Faculty of Pharmacy, Helwan University, Cairo, Egypt.Data acquisition for quantification and confirmation are performed in full scan negative ion mode. Samples are individually tuned for each target analyzes by direct injectionof the individual solution $(1 \mathrm{mg} / \mathrm{mL})$. Data acquisition and processing is performed using Thermo Scientific Xcalibur 2.1 software. Microanalyses were operated using Vario, Elmentar apparatus (Shimadzu, Japan), Organic Microanalysis Unit, Cairo University, Giza, Egypt. Column chromatography was performed on silica gel 60 (particle size $0.06 \mathrm{~mm}-0.20 \mathrm{~mm}$ ). All compounds prepared in this paper are new except (1d) [20], and confirmed with spectral data. Hippuric acid and oxazolone 3 were prepared according to standard procedures and their structure confirmation data were found identical to literature [12].

\subsection{General Procedure for Preparation of Vinyl Peptide 1a-q}

In the microwave reaction tube, $5 \mathrm{mmol}$ of both oxazolone 3 and the appropriate amine were placed and 5 $\mathrm{mL}$ of $\mathrm{N}, \mathrm{N}$-dimethylformamide (DMF) was added. The reaction was heated to $200^{\circ} \mathrm{C}$ while stirring in Milestone 
microwave reactor (SynthLab) for 10 minutes. After cooling, the mixture was added slowly to dilute $\mathrm{HCl}$ mixed with crushed ice. The resulting solid was collected by filtration, washed with water and purified using silica gel chromatography (petroleum ether $/ \mathrm{CHCl}_{3} / \mathrm{EtOH}$, gradient).

\subsubsection{2-(Benzoylamino)-3-(2-Furyl)- $N$ - Ethylpropenamide (1a) [20]}

White solid, mp: $133^{\circ} \mathrm{C}-134^{\circ} \mathrm{C} .{ }^{1} \mathrm{H}$ NMR $(600 \mathrm{MHz}$, $\left.\mathrm{CDCl}_{3}\right) \delta: 8.48(1 \mathrm{H}, \mathrm{s}, \mathrm{NH}), 7.96(2 \mathrm{H}, \mathrm{d}, J=7.2 \mathrm{~Hz}$, ArH), $7.60(1 \mathrm{H}, \mathrm{t}, J=7.2 \mathrm{~Hz}, \mathrm{ArH}), 7.51(2 \mathrm{H}, \mathrm{t}, J=7.2$ $\mathrm{Hz}, \mathrm{ArH}), 7.45$ ( $1 \mathrm{H}, \mathrm{s}, \mathrm{ArH}), 6.89(1 \mathrm{H}, \mathrm{s}, \mathrm{HC}=\mathrm{C}), 6.52$ $(1 \mathrm{H}, \mathrm{d}, J=2.4 \mathrm{~Hz}, \mathrm{ArH}), 6.46(2 \mathrm{H}, \mathrm{s}, \mathrm{ArH}$ and $\mathrm{NH}), 3.45$ $\left(2 \mathrm{H}, \mathrm{q}, J=6.6 \mathrm{~Hz}, \mathrm{CH}_{2}\right), 0.95\left(3 \mathrm{H}, \mathrm{t}, J=7.2 \mathrm{~Hz}, \mathrm{CH}_{3}\right)$. IR $\left(\mathrm{KBr}, v, \mathrm{~cm}^{-1}\right): 3162,3053,3018,2940,1687,1636$, 1620. MS: RT $0.58 \mathrm{~min}, m / z 284$ (M, 40\%), 268 (100\%).

\subsubsection{2-(Benzoylamino)-3-(2-Furyl)- $N$ - Cyclopentylpropenamide (1b)}

White solid, mp: $169^{\circ} \mathrm{C}-171^{\circ} \mathrm{C} .{ }^{1} \mathrm{H}$ NMR $(600 \mathrm{MHz}$, DMSO-d6) $\delta: 9.71(1 \mathrm{H}, \mathrm{s}, \mathrm{NH}), 8.03(2 \mathrm{H}, \mathrm{d}, J=7.2 \mathrm{~Hz}$, ArH), $7.93(1 \mathrm{H}, \mathrm{d}, J=6.6 \mathrm{~Hz}, \mathrm{ArH}), 7.73(1 \mathrm{H}, \mathrm{d}, J=6.6$ $\mathrm{Hz}, \mathrm{ArH}), 7.60(1 \mathrm{H}, \mathrm{br}, \mathrm{NH}), 7.53(2 \mathrm{H}, \mathrm{t}, J=6.6 \mathrm{~Hz}$, $\mathrm{ArH}), 7.05(1 \mathrm{H}, \mathrm{s}, \mathrm{HC}=\mathrm{C}), 6.66(1 \mathrm{H}, \mathrm{d}, J=2.4 \mathrm{~Hz})$, $6.56(1 \mathrm{H}, \mathrm{s}, \mathrm{HC}=\mathrm{C}), 3.45(2 \mathrm{H}, \mathrm{q}, J=6.6 \mathrm{~Hz} \mathrm{CH}-\mathrm{Cy}-$ lopentyl), 1.82 (2H, m, CH-cyclopentyl), $1.65(2 \mathrm{H}, \mathrm{m}$, $\mathrm{CH}$-cyclopentyl), 1.50 (4H, m, CH-cyclopentyl). IR ( $\mathrm{KBr}$, $\left.v, \mathrm{~cm}^{-1}\right): 3162,3053,3018,3005,2937,1676,1631$, 1623. MS: RT $0.23 \mathrm{~min}, \mathrm{~m} / z 323$ (M-1, 60\%), 298 $(100 \%)$.

\subsubsection{2-(Benzoylamino)-3-(2-Furyl)- $N$-(1-Adamantyl) Propenamide (1c)}

White solid, mp: $185^{\circ} \mathrm{C}-187^{\circ} \mathrm{C} .{ }^{1} \mathrm{H}$ NMR $(600 \mathrm{MHz}$, DMSO-d6) $\delta: 9.71(1 \mathrm{H}, \mathrm{s}, \mathrm{NH}), 8.02(2 \mathrm{H}, \mathrm{d}, J=6.0 \mathrm{~Hz}$, $\mathrm{ArH}), 7.74$ (1H, s, ArH), $7.61(1 \mathrm{H}, \mathrm{t}, J=6.6 \mathrm{~Hz}, \mathrm{ArH})$, $7.54(2 \mathrm{H}, \mathrm{t}, J=7.2 \mathrm{~Hz}, \mathrm{ArH}), 7.11(1 \mathrm{H}, \mathrm{s}, \mathrm{HC}=\mathrm{CH})$, $6.99(1 \mathrm{H}, \mathrm{s}, \mathrm{NH}), 6.66(1 \mathrm{H}, \mathrm{d}, J=3.0 \mathrm{~Hz}), 6.56(1 \mathrm{H}, \mathrm{s}$, ArH), 3.33 (1H, m, CH-adamanty), 2.03 (3H, m, CH-adamantyl), 1.99 (6H, m, CH-adamantyl), 1.64 (7H, m, $\mathrm{CH}$-adamantyl). IR (KBr, v, $\left.\mathrm{cm}^{-1}\right)$ : 3146, 3048, 3030, 2952 - 3926, 1662, 1641, 1618. Anal.Calcd. For $\mathrm{C}_{24} \mathrm{H}_{26} \mathrm{~N}_{2} \mathrm{O}_{3}$ : C, 73.82; H, 6.71; N, 7.17. Found: C, 74.20; $\mathrm{H}, 6.73$; N, 7.32. MS: RT $0.85 \mathrm{~min}, \mathrm{~m} / \mathrm{z} 389$ (M-1, 80\%), $276(100 \%)$.

\subsubsection{2-(Benzoylamino)-3-(2-Furyl)- $N$ - Phenylpropenamide (1d)}

White solid, mp: $192^{\circ} \mathrm{C}-194^{\circ} \mathrm{C} .{ }^{1} \mathrm{H}$ NMR $(600 \mathrm{MHz}$, DMSO-d6) $\delta: 10.06(1 \mathrm{H}, \mathrm{s}, \mathrm{NH}), 9.96(1 \mathrm{H}, \mathrm{s}, \mathrm{NH}), 8.08$ $(2 \mathrm{H}, \mathrm{d}, J=6.6 \mathrm{~Hz}, \mathrm{ArH}), 7.81(1 \mathrm{H}, \mathrm{s}), 7.72(2 \mathrm{H}, \mathrm{d}, J=$ $7.2 \mathrm{~Hz}, \mathrm{ArH}), 7.62(1 \mathrm{H}, \mathrm{d}, J=6.6 \mathrm{~Hz}, \mathrm{ArH}), 7.56(2 \mathrm{H}$, $\mathrm{d}, J=6.6 \mathrm{~Hz}, \mathrm{ArH}), 7.33$ ( $2 \mathrm{H}, \mathrm{t}, J=7.2, \mathrm{ArH}), 7.14$
$(1 \mathrm{H}, \mathrm{s}, \mathrm{HC}=\mathrm{C}), 7.08(1 \mathrm{H}, \mathrm{t}, J=6.6, \mathrm{ArH}), 6.78(1 \mathrm{H}, \mathrm{d}$, $J=3.0 \mathrm{~Hz}, \mathrm{ArH}), 6.62(1 \mathrm{H}, \mathrm{s}, \mathrm{ArH}) . \mathrm{IR}\left(\mathrm{KBr}, v, \mathrm{~cm}^{-1}\right)$ : 3162, 3053, 3018, 1687, 1636, 1620. Anal.Calcd. For $\mathrm{C}_{20} \mathrm{H}_{16} \mathrm{~N}_{2} \mathrm{O}_{3}$ : C, 72.28; H, 4.85; N, 8.43. Found: C, 72.48; H, 5.11; N, 8.32. MS: RT $0.32 \mathrm{~min}, \mathrm{~m} / \mathrm{z} 331$ (M-1, 33\%), 298 $(100 \%)$.

\subsubsection{2-(Benzoylamino)-3-(2-Furyl)- $N$ - (3-Methylphenyl) Propenamide (1e)}

Off-white solid, mp: $162^{\circ} \mathrm{C}-163^{\circ} \mathrm{C} .{ }^{1} \mathrm{H}$ NMR $(600 \mathrm{MHz}$, $\left.\mathrm{CDCl}_{3}\right) \delta: 8.59(2 \mathrm{H}, \mathrm{s}, \mathrm{NH}), 7.99(2 \mathrm{H}, \mathrm{d}, J=7.8 \mathrm{~Hz}$, ArH), $7.61(1 \mathrm{H}, \mathrm{t}, J=7.2 \mathrm{~Hz}, \mathrm{ArH}), 7.52(2 \mathrm{H}, \mathrm{t}, J=7.2$ $\mathrm{Hz}, \mathrm{ArH}), 7.47$ (1H, s, ArH), 7.43 (1H, s, ArH), $7.39(1 \mathrm{H}$, $\mathrm{d}, J=7.8 \mathrm{~Hz}, \operatorname{ArH}), 7.18(1 \mathrm{H}, \mathrm{t}, J=7.8 \mathrm{~Hz}, \mathrm{ArH}), 6.97$ $(1 \mathrm{H}, \mathrm{s}, \mathrm{HC}=\mathrm{C}), 6.91(1 \mathrm{H}, \mathrm{d}, J=7.2 \mathrm{~Hz}, \mathrm{ArH}), 6.51(1 \mathrm{H}$, $\mathrm{s}, \mathrm{ArH}), 6.47$ (1H, s, ArH), 2.32 (3H, s, CH3). IR (KBr, v, $\left.\mathrm{cm}^{-1}\right): 3155,3092,3011,2931,1665,1621,1618 . \mathrm{MS}$ : RT $0.59 \mathrm{~min}, \mathrm{~m} / \mathrm{z} 345$ (M-1, 25\%), 265 (100\%).

\subsubsection{2-(Benzoylamino)-3-(2-Furyl)- $N$ - (4-Methylphenyl) Propenamide (1f)}

Pale brown solid, mp: $182^{\circ} \mathrm{C}-184^{\circ} \mathrm{C}$. ${ }^{1} \mathrm{H}$ NMR $(600$ $\left.\mathrm{MHz} \mathrm{CDCl}_{3}\right) \delta: 8.56(2 \mathrm{H}, \mathrm{br}, \mathrm{NH}), 7.98(2 \mathrm{H}, \mathrm{d}, J=6.0$ $\mathrm{Hz}, \mathrm{ArH}), 7.60(1 \mathrm{H}, \mathrm{t}, J=6.6 \mathrm{~Hz}, \mathrm{ArH}), 7.53(2 \mathrm{H}, \mathrm{d}, J$ $=7.2 \mathrm{~Hz}, \mathrm{ArH}), 7.46(2 \mathrm{H}, \mathrm{d}, \mathrm{J}=6.6 \mathrm{~Hz}, \mathrm{ArH}), 7.10$ $(2 \mathrm{H}, \mathrm{d}, J=7.2 \mathrm{~Hz}, \mathrm{ArH}), 6.99(1 \mathrm{H}, \mathrm{s}, \mathrm{HC}=\mathrm{C}), 6.52$ (1H, s, ArH), $6.46(1 \mathrm{H}, \mathrm{s}, \mathrm{ArH}), 2.30\left(3 \mathrm{H}, \mathrm{s}, \mathrm{CH}_{3}\right) . \mathrm{IR}$ $\left(\mathrm{KBr}, v, \mathrm{~cm}^{-1}\right): 3154,3096,3016,2935,1669,1629$, 1617. MS: RT $0.76 \mathrm{~min}, \mathrm{~m} / z 345$ (M-1, 15\%), 279 $(100 \%)$.

\subsubsection{2-(Benzoylamino)-3-(2-Furyl)- $N$ - (3-Fluorophenyl) Propenamide (1g)}

White solid, mp: $217^{\circ} \mathrm{C}-218^{\circ} \mathrm{C} .{ }^{1} \mathrm{H}$ NMR $(600 \mathrm{MHz}$, DMSO-d6) $\delta: 10.26$ (1H, s, NH), 9.99 (1H, s, NH), 8.07 $(2 \mathrm{H}, \mathrm{d}, J=7.2 \mathrm{~Hz}, \mathrm{ArH}), 7.82(1 \mathrm{H}, \mathrm{s}), 7.70(1 \mathrm{H}, \mathrm{d}, J=$ $12 \mathrm{~Hz}, \mathrm{ArH}), 7.61(1 \mathrm{H}, \mathrm{t}, J=7.2 \mathrm{~Hz}, \operatorname{ArH}), 7.56(2 \mathrm{H}, \mathrm{t}, J=$ $7.2 \mathrm{~Hz}, \mathrm{ArH}), 7.52(1 \mathrm{H}, \mathrm{d}, J=7.8 \mathrm{~Hz}, \mathrm{ArH}), 7.36(1 \mathrm{H}$, $\mathrm{dd}, J=7.2$ and $15.0 \mathrm{~Hz}, \mathrm{ArH}), 7.13(1 \mathrm{H}, \mathrm{s}, \mathrm{HC}=\mathrm{C})$, $6.91(1 \mathrm{H}, \mathrm{t}, J=7.2 \mathrm{~Hz}, \operatorname{ArH}), 6.80(1 \mathrm{H}, \mathrm{d}, J=3.0 \mathrm{~Hz}$, ArH), 6.63 (1H, s, ArH). IR (KBr, v, $\left.\mathrm{cm}^{-1}\right): 3162,3041$, $3015,1678,1621,1618$.

\subsubsection{2-(Benzoylamino)-3-(2-Furyl)- $N$ - (4-Fluorophenyl) Propenamide (1h)}

White solid, mp: $200^{\circ} \mathrm{C}-202^{\circ} \mathrm{C} .{ }^{1} \mathrm{H}$ NMR $(600 \mathrm{MHz}$, DMSO-d6) $\delta$ : $10.12(1 \mathrm{H}, \mathrm{s}, \mathrm{NH}), 9.97(1 \mathrm{H}, \mathrm{s}, \mathrm{NH}), 8.08$ $(2 \mathrm{H}, \mathrm{d}, J=6.6 \mathrm{~Hz}, \mathrm{ArH}), 7.81(1 \mathrm{H}, \mathrm{s}), 7.74(1 \mathrm{H}, \mathrm{dd}, J=$ 3.6 and $4.8 \mathrm{~Hz}, \mathrm{ArH}), 7.62(1 \mathrm{H}, \mathrm{d}, J=6.6 \mathrm{~Hz}, \mathrm{ArH}), 7.56$ $(2 \mathrm{H}, \mathrm{t}, J=6.6 \mathrm{~Hz}, \mathrm{ArH}), 7.17(2 \mathrm{H}, \mathrm{t}, J=7.2 \mathrm{~Hz}, \mathrm{ArH})$ $7.14(1 \mathrm{H}, \mathrm{s}, \mathrm{HC}=\mathrm{C}), 6.79(1 \mathrm{H}, \mathrm{d}, J=3.0 \mathrm{~Hz}, \mathrm{ArH}), 6.62$ $(1 \mathrm{H}, \mathrm{s}, \mathrm{Ar})$. IR (KBr, $\left.v, \mathrm{~cm}^{-1}\right): 3160,3039,3015,1675$, 1620, 1618. MS: RT $0.66 \mathrm{~min}, \mathrm{~m} / z 350$ (M, 100\%), 281 $(65 \%)$. 


\subsubsection{2-(Benzoylamino)-3-(2-Furyl)- $N$ -}

(3-Chlorophenyl) Propenamide (1i)

Off-white solid, mp: $206^{\circ} \mathrm{C}-208^{\circ} \mathrm{C} .{ }^{1} \mathrm{H}$ NMR $(600 \mathrm{MHz}$, DMSO-d6) $\delta: 10.23(1 \mathrm{H}, \mathrm{s}, \mathrm{NH}), 9.99(1 \mathrm{H}, \mathrm{s}, \mathrm{NH}), 8.07$ $(2 \mathrm{H}, \mathrm{d}, J=7.2 \mathrm{~Hz}, \mathrm{ArH}), 7.91(1 \mathrm{H}, \mathrm{s}), 7.82(1 \mathrm{H}, \mathrm{s}, \mathrm{ArH})$, $7.68(1 \mathrm{H}, \mathrm{d}, J=6.0 \mathrm{~Hz}, \mathrm{ArH}), 7.62(1 \mathrm{H}, \mathrm{d}, \mathrm{J}=6.0 \mathrm{~Hz}$, $\mathrm{ArH}), 7.56(2 \mathrm{H}, \mathrm{t}, J=6.0 \mathrm{~Hz}, \mathrm{ArH}), 7.36(1 \mathrm{H}, \mathrm{t}, J=6.0$ $\mathrm{Hz}, \mathrm{ArH}), 7.14(2 \mathrm{H}, \mathrm{m}, \mathrm{ArH}$ and $\mathrm{HC}=\mathrm{C}), 6.81(1 \mathrm{H}, \mathrm{d}, J=$ $6.0 \mathrm{~Hz}, \mathrm{ArH}), 6.63(1 \mathrm{H}, \mathrm{s}, \mathrm{ArH}) . \mathrm{IR}\left(\mathrm{KBr}, v, \mathrm{~cm}^{-1}\right): 3166$, 3032, 3018, 1684, 1620, 1613. MS: RT $0.58 \mathrm{~min}, \mathrm{~m} / \mathrm{z}$ 365 (M-1, 25\%), 363 (100\%).

\subsubsection{2-(Benzoylamino)-3-(2-Furyl)- $N$ - (3-Methoxyphenyl) Propenamide (1j)}

White solid, mp: $160^{\circ} \mathrm{C}-161^{\circ} \mathrm{C} .{ }^{1} \mathrm{H}$ NMR $(600 \mathrm{MHz}$, $\left.\mathrm{CDCl}_{3}\right) \delta: 8.59(1 \mathrm{H}, \mathrm{br}, \mathrm{NH}), 8.64(1 \mathrm{H}, \mathrm{br}, \mathrm{NH}), 7.95$ $(2 \mathrm{H}, \mathrm{d}, J=7.2 \mathrm{~Hz}, \mathrm{ArH}), 7.57(1 \mathrm{H}, \mathrm{t}, J=7.2 \mathrm{~Hz}, \mathrm{ArH})$, $7.47(2 \mathrm{H}, \mathrm{t}, J=7.2 \mathrm{~Hz}, \mathrm{ArH}), 7.38(1 \mathrm{H}, \mathrm{s}, \mathrm{ArH}), 7.38$ $(1 \mathrm{H}, \mathrm{s}, \mathrm{ArH}), 7.34(1 \mathrm{H}, \mathrm{s}, \mathrm{ArH}), 7.12(1 \mathrm{H}, \mathrm{t}, J=7.8 \mathrm{~Hz}$, $\mathrm{ArH}), 7.08(1 \mathrm{H}, \mathrm{d}, J=7.8 \mathrm{~Hz}, \mathrm{ArH}), 6.80(1 \mathrm{H}, \mathrm{s}, \mathrm{HC}=$ C), $6.59(1 \mathrm{H}, \mathrm{dd}, J=7.8$ and $1.8 \mathrm{~Hz}, \mathrm{ArH}), 6.38(2 \mathrm{H}, \mathrm{d}, J=$ $7.2 \mathrm{~Hz}, \mathrm{ArH}), 3.76\left(3 \mathrm{H}, \mathrm{s}, \mathrm{OCH}_{3}\right)$. IR $\left(\mathrm{KBr}, v, \mathrm{~cm}^{-1}\right)$ : 3160, 3039, 3015, 1675, 1620, 1618. Anal.Calcd. For $\mathrm{C}_{21} \mathrm{H}_{18} \mathrm{~N}_{2} \mathrm{O}_{4}$ : C, 69.60; H, 5.01; N, 7.73; O, 17.66. Found: C, 69.59; H, 5.13; N, 7.89.

\subsubsection{2-(Benzoylamino)-3-(2-Furyl)- $N$ - (3-Hydroxyphenyl) Propenamide (1k)}

White solid, mp: $110^{\circ} \mathrm{C}-112^{\circ} \mathrm{C} .{ }^{1} \mathrm{H}$ NMR $(600 \mathrm{MHz}$, DMSO-d6) $\delta: 9.45(1 \mathrm{H}, \mathrm{s}, \mathrm{NH}), 9.31(1 \mathrm{H}, \mathrm{s}, \mathrm{NH}), 8.90$ $(1 \mathrm{H}, \mathrm{s}, \mathrm{OH}), 8.06(2 \mathrm{H}, \mathrm{m}, \mathrm{ArH}), 7.63(1 \mathrm{H}, \mathrm{d}, J=7.2 \mathrm{~Hz}$, $\mathrm{ArH}), 7.58(1 \mathrm{H}, \mathrm{d}, J=6.6 \mathrm{~Hz}, \mathrm{ArH}), 7.51(2 \mathrm{H}, \mathrm{d}, J=7.2$ $\mathrm{Hz}, \mathrm{ArH}), 7.30(1 \mathrm{H}, \mathrm{s}, \mathrm{ArH}), 7.18(1 \mathrm{H}, \mathrm{s}, \mathrm{ArH}), 7.05(2 \mathrm{H}$, $\mathrm{m}, \operatorname{ArH}), 6.64(1 \mathrm{H}, \mathrm{s}, \mathrm{HC}=\mathrm{C}), 6.53(1 \mathrm{H}, \mathrm{d}, J=7.2$, $\mathrm{ArH}), 6.48(1 \mathrm{H}, \mathrm{d}, J=7.8 \mathrm{~Hz}, \mathrm{ArH})$. IR $\left(\mathrm{KBr}, v, \mathrm{~cm}^{-1}\right)$ : $3360,3160,3039,3015,1675,1618$. Anal.Calcd. For $\mathrm{C}_{20} \mathrm{H}_{16} \mathrm{~N}_{2} \mathrm{O}_{4}$ : C, 68.96; H, 4.63; N, 8.04. Found: C, 68.59; $\mathrm{H}, 4.32 ; \mathrm{N}, 7.98$.

\subsubsection{2-(Benzoylamino)-3-(2-Furyl)- $\mathrm{N}$ - (4-Aminosulfonylphenyl) Propenamide (11)}

White solid, mp: $140^{\circ} \mathrm{C}-143^{\circ} \mathrm{C} .{ }^{1} \mathrm{H}$ NMR $(600 \mathrm{MHz}$, DMSO-d6) $\delta: 10.35(1 \mathrm{H}, \mathrm{s}, \mathrm{NH}), 9.98(1 \mathrm{H}, \mathrm{s}, \mathrm{NH}), 8.05$ $(2 \mathrm{H}, \mathrm{d}, J=7.2 \mathrm{~Hz}, \mathrm{ArH}), 7.87(2 \mathrm{H}, \mathrm{d}, J=7.8 \mathrm{~Hz}, \mathrm{ArH})$, $7.80(1 \mathrm{H}, \mathrm{d}, J=1.2 \mathrm{~Hz}, \mathrm{ArH}), 7.76(1 \mathrm{H}, \mathrm{s}, \mathrm{ArH}), 7.74$ $(1 \mathrm{H}, \mathrm{s}, \mathrm{ArH}), 7.60(1 \mathrm{H}, \mathrm{t}, J=7.2 \mathrm{~Hz}, \mathrm{ArH}), 7.53(2 \mathrm{H}, \mathrm{t}, J=$ $7.2 \mathrm{~Hz}, \mathrm{ArH}), 7.24\left(2 \mathrm{H}, \mathrm{s}, \mathrm{SO}_{2} \mathrm{NH}_{2}\right), 7.13(1 \mathrm{H}, \mathrm{s}, \mathrm{HC}=$ C), $6.78(1 \mathrm{H}, \mathrm{d}, J=3.6 \mathrm{~Hz}, \mathrm{ArH}), 6.60(1 \mathrm{H}, \mathrm{dd}, J=3.6$ and $1.8 \mathrm{~Hz}, \mathrm{ArH})$. IR (KBr, $\left.v, \mathrm{~cm}^{-1}\right): 3510,3390,3352$, $3160,3039,3029,1681,1622$.

\subsubsection{2-(Benzoylamino)-3-(2-Furyl)- $N$ -} (4-Nitrophenyl) Propenamide (1m)

Off-white solid, mp: $229^{\circ} \mathrm{C}-230^{\circ} \mathrm{C} .{ }^{1} \mathrm{H}$ NMR $(600 \mathrm{MHz}$,
DMSO-d6) $\delta: 10.67(1 \mathrm{H}, \mathrm{s}, \mathrm{NH}), 10.06(1 \mathrm{H}, \mathrm{s}, \mathrm{NH}), 8.25$ $(2 \mathrm{H}, \mathrm{dd}, J=7.2$ and $2.4 \mathrm{~Hz}, \mathrm{ArH}), 8.07(2 \mathrm{H}, \mathrm{m}, \mathrm{ArH})$, $8.01(2 \mathrm{H}, \mathrm{dd}, J=7.8$ and $2.4 \mathrm{~Hz}, \mathrm{ArH}), 7.85(1 \mathrm{H}, \mathrm{s}, \mathrm{ArH})$, $7.63(1 \mathrm{H}, \mathrm{s}, \mathrm{ArH}), 7.56(2 \mathrm{H}, \mathrm{s}, \mathrm{ArH}), 7.16(1 \mathrm{H}, \mathrm{s}, \mathrm{HC}=$ C), $6.85(1 \mathrm{H}, \mathrm{d}, J=3.0 \mathrm{~Hz}, \mathrm{ArH}), 6.65(1 \mathrm{H}, \mathrm{s}, \mathrm{ArH})$. IR $\left(\mathrm{KBr}, v, \mathrm{~cm}^{-1}\right)$ : 3307, 3279, 3220, 3096, 1685, 1619, 1598, 1507, 1344.

\subsubsection{2-(Benzoylamino)-3-(2-Furyl)- $N$ - (4-Fluorobenzyl) Propenamide (1n)}

White solid, mp: $128^{\circ} \mathrm{C} .{ }^{1} \mathrm{H}$ NMR (600 MHz, DMSO-d6) $\delta: 9.85(1 \mathrm{H}, \mathrm{s}, \mathrm{NH}), 8.68(1 \mathrm{H}, \mathrm{br}, \mathrm{NH}), 8.07(2 \mathrm{H}, \mathrm{d}, J=$ $7.2 \mathrm{~Hz}, \mathrm{ArH}), 7.75(1 \mathrm{H}, \mathrm{d}, J=1.2 \mathrm{~Hz}, \mathrm{ArH}), 7.61(1 \mathrm{H}, \mathrm{t}$, $J=7.2 \mathrm{~Hz}, \operatorname{ArH}), 7.54(2 \mathrm{H}, \mathrm{t}, J=7.2 \mathrm{~Hz}, \operatorname{ArH}), 7.35(2 \mathrm{H}$, $\mathrm{t}, J=7.2 \mathrm{~Hz}, \mathrm{ArH}), 7.22(1 \mathrm{H}, \mathrm{s}, \mathrm{HC}=\mathrm{C}), 7.14(2 \mathrm{H}, \mathrm{t}, J=$ $7.8 \mathrm{~Hz}, \mathrm{ArH}), 6.71(1 \mathrm{H}, \mathrm{d}, J=13.0 \mathrm{~Hz}, \mathrm{ArH}), 6.57(1 \mathrm{H}$, dd, $J=3$ and $1.8 \mathrm{~Hz}, \mathrm{ArH}), 4.36(2 \mathrm{H}, \mathrm{s})$. IR (KBr, $v$, $\left.\mathrm{cm}^{-1}\right): 3132,3046,3032,2937,1632,1617$. MS: RT 0.78 min, $m / z 363$ (M-1, 47\%), 284 (100\%).

\subsubsection{2-(Benzoylamino)-3-(2-Furyl)- $N$-[2- (5-Cyanothiazol-2-Yl)] Propenamide (10)}

Yellow-white solid, mp: $198^{\circ} \mathrm{C}-199^{\circ} \mathrm{C} .{ }^{1} \mathrm{H}$ NMR $(600$ MHz, DMSO-d6) $\delta: 13.32(1 \mathrm{H}, \mathrm{s}, \mathrm{NH}), 10.10(1 \mathrm{H}, \mathrm{s}$, $\mathrm{NH}), 8.43(1 \mathrm{H}, \mathrm{s}, \mathrm{ArH}), 8.08(2 \mathrm{H}, \mathrm{d}, J=6.6), 7.87(1 \mathrm{H}$, $\mathrm{s}), 7.63(\mathrm{~m}, 2 \mathrm{H}, \mathrm{ArH}), 7.56(2 \mathrm{H}, \mathrm{d}, J=6.6 \mathrm{~Hz}, \mathrm{ArH})$, $7.38(1 \mathrm{H}, \mathrm{s}, \mathrm{HC}=\mathrm{C}), 6.9(1 \mathrm{H}, \mathrm{s}, \mathrm{ArH}), 6.65(\mathrm{~s}, 1 \mathrm{H}, \mathrm{ArH})$. Anal.Calcd. For $\mathrm{C}_{18} \mathrm{H}_{12} \mathrm{~N}_{4} \mathrm{O}_{3} \mathrm{~S}$ : C, 59.33; H, 3.32; N, 15.38; O, 13.17; S, 8.80. Found: C, 58.99; H, 3.52; N, 15.37. MS: RT $0.78 \mathrm{~min}, \mathrm{~m} / \mathrm{z} 363$ (M-1, 25\%), 275 (100\%).

\subsubsection{2-(Benzoylamino)-3-(2-Furyl)- $N$-(2-Pyridyl) Propenamide (1p)}

Pale Brown solid, mp: $160^{\circ} \mathrm{C} .{ }^{1} \mathrm{H}$ NMR $(600 \mathrm{MHz}$, $\left.\mathrm{CDCl}_{3}\right) \delta: 8.83(1 \mathrm{H}, \mathrm{s}, \mathrm{NH}), 8.59(1 \mathrm{H}, \mathrm{s}, \mathrm{NH}), 8.32(1 \mathrm{H}$, $\mathrm{d}, J=7.2 \mathrm{~Hz}, \mathrm{ArH}), 8.24(1 \mathrm{H}, \mathrm{d}, J=4.2 \mathrm{~Hz}, \mathrm{ArH}), 7.97$ $(2 \mathrm{H}, \mathrm{d}, J=6.6 \mathrm{~Hz}, \mathrm{ArH}), 7.70(1 \mathrm{H}, \mathrm{t}, J=7.2 \mathrm{~Hz}), 7.59$ $(1 \mathrm{H}, \mathrm{t}, J=7.2 \mathrm{~Hz}), 7.52(3 \mathrm{H}, \mathrm{m}, \mathrm{ArH}), 7.06(1 \mathrm{H}, \mathrm{s}, \mathrm{HC}=$ C), $7.01(1 \mathrm{H}, \mathrm{t}, J=6.6 \mathrm{~Hz}, \mathrm{ArH}), 6.60(1 \mathrm{H}, \mathrm{d}, J=3.6 \mathrm{~Hz}$, $\mathrm{ArH}), 6.49(1 \mathrm{H}, \mathrm{t}, J=1.8$ and $1.2 \mathrm{~Hz}, \mathrm{ArH})$. IR (KBr, $v$, $\left.\mathrm{cm}^{-1}\right)$ : 3169, 3055, 3026, 1692, 1600, 1560. MS: RT 0.44 $\mathrm{min}, \mathrm{m} / \mathrm{z} 332$ (M-1, 55\%), 284 (100\%).

\subsubsection{2-(Benzoylamino)-3-(2-Furyl)- $N$-(3-Pyridyl) Propenamide (1q)}

${ }^{1} \mathrm{H}$ NMR $\left(600 \mathrm{MHz}, \mathrm{CDCl}_{3}\right) \delta: 9.05(1 \mathrm{H}, \mathrm{s}, \mathrm{NH}), 8.67$ $(2 \mathrm{H}, \mathrm{s}, \mathrm{NH}$ and $\mathrm{ArH}), 8.31(1 \mathrm{H}, \mathrm{d}, J=3.6 \mathrm{~Hz}, \mathrm{ArH}), 8.18$ $(1 \mathrm{H}, \mathrm{d}, J=7.2 \mathrm{~Hz}), 7.98(2 \mathrm{H}, \mathrm{d}, J=7.2 \mathrm{~Hz}, \mathrm{ArH}), 7.61$ $(1 \mathrm{H}, \mathrm{t}, J=7.2 \mathrm{~Hz}), 7.52(2 \mathrm{H}, \mathrm{d}, J=7.2 \mathrm{~Hz}, \mathrm{ArH}), 7.47$ $(1 \mathrm{H}, \mathrm{d}, J=6.6 \mathrm{~Hz}, \mathrm{ArH}), 7.22(1 \mathrm{H}, \mathrm{t}, J=7.2 \mathrm{~Hz}), 6.98$ $(1 \mathrm{H}, \mathrm{s}, \mathrm{HC}=\mathrm{C}), 6.60(1 \mathrm{H}, \mathrm{d}, J=3.0 \mathrm{~Hz}, \mathrm{ArH}), 6.47(1 \mathrm{H}$, s, ArH). IR (KBr, v, cm $\left.{ }^{-1}\right): 3159,3061,3028,1694,1598$, 
1558. MS: RT $0.31 \mathrm{~min}, \mathrm{~m} / \mathrm{z} 332$ (M-1, 15\%), 329 $(100 \%)$.

\subsubsection{4-[(Furan-2-yl) Methylene]-1- (4-Hydroxyphenyl)-2-Phenyl-1H-Imidazol- 5(4H)-One (4)}

${ }^{1} \mathrm{H}$ NMR $\left(600 \mathrm{MHz}, \mathrm{CDCl}_{3}\right) \delta: 7.67(1 \mathrm{H}, \mathrm{s}, \mathrm{ArH}), 7.59$ $(2 \mathrm{H}, \mathrm{d}, J=7.2 \mathrm{~Hz}, \mathrm{ArH}), 7.56(1 \mathrm{H}, \mathrm{s}, \mathrm{HC}=\mathrm{C}), 7.41(1 \mathrm{H}$, $\mathrm{t}, J=7.2 \mathrm{~Hz}, \mathrm{ArH}), 7.30(2 \mathrm{H}, \mathrm{t}, J=7.2 \mathrm{~Hz}, \mathrm{ArH}), 7.20$ $(1 \mathrm{H}, \mathrm{s}, \mathrm{OH}), 6.97(2 \mathrm{H}, \mathrm{d}, J=8.4 \mathrm{~Hz}, \mathrm{ArH}), 6.75(2 \mathrm{H}, \mathrm{d}, J=$ $8.4 \mathrm{~Hz}, \operatorname{ArH}), 6.63(1 \mathrm{H}, \mathrm{s}, \mathrm{ArH}), 6.45(1 \mathrm{H}, \mathrm{s}, \mathrm{ArH}) . \mathrm{IR}$ $\left(\mathrm{KBr}, v, \mathrm{~cm}^{-1}\right): 3360,3084,1745,1622,1578 . \mathrm{LC} / \mathrm{MS}$ : LC/MS: Rt $18.65 \mathrm{~min}, \mathrm{~m} / \mathrm{z} 331(\mathrm{M}+1,100 \%)$. Anal.Calcd. For $\mathrm{C}_{20} \mathrm{H}_{14} \mathrm{~N}_{2} \mathrm{O}_{3}$ : C, 72.72; H, 4.27; N, 8.48. Found: C, 72.88; H, 3.91; N, 8.71. MS: RT $0.2 \mathrm{~min}, \mathrm{~m} / \mathrm{z}$ $330(\mathrm{M}, 100 \%)$.

\subsection{MCF-7 Cancer Cell Line Inhibition Test}

\subsubsection{Cell Culture}

MCF-7 human breast cancer cells was grown in RPMI1640 medium, supplemented with $10 \%$ heat inactivated FBS, 50 units $/ \mathrm{mL}$ of penicillin and $50 \mathrm{mg} / \mathrm{mL}$ of streptomycin and maintained at $37^{\circ}$ in a humidified atmosphere containing $5 \% \mathrm{CO}_{2}$. The cells were maintained as "monolayer culture" by serial subculturing.

\subsubsection{SRB Cytotoxicity Assay}

Exponentially growing cells were collected using $0.25 \%$ Trypsin-EDTA and seeded in 96-well plates at 1000 2000 cells/well in RPMI-1640 supplemented medium. After $24 \mathrm{~h}$, cells were incubated for $72 \mathrm{~h}$ with various concentrations of the tested compounds. Following $72 \mathrm{~h}$ treatment, the cells will be fixed with $10 \%$ trichloroacetic acid for $1 \mathrm{~h}$ at $4^{\circ} \mathrm{C}$. Wells were stained for $10 \mathrm{~min}$ at room temperature with $0.4 \%$ SRB dissolved in $1 \%$ acetic acid. The plates were air dried for $24 \mathrm{~h}$ and the dye was solubilized with Tris- $\mathrm{HCl}$ for $5 \mathrm{~min}$ on a shaker at 1600 rpm. The optical density (OD) of each well was measured spectrophotometrically at $564 \mathrm{~nm}$ with an ELISA microplate reader (ChroMate-4300, FL, USA). The IC50 values were calculated according to the equation for Boltzman sigmoidal concentration-response curve using the nonlinear regression fitting models (Graph Pad, Prism Version 5).

\subsection{Chimney Test for Neurotoxicity}

The animals had to climb backwards up a glass tube (3 $\mathrm{cm}$ inner diameter, $25 \mathrm{~cm}$ long). Motor impairment was evidenced by the inability of mice to climb backwards up the tube within $30 \mathrm{~s}$. Mice were divided into groups of 6 animals each. The first group received vehicle (tween $80 /$ saline) and it had no neurotoxic symptoms. The rest of Groups was given the tested compound at $0.15 \mathrm{M}$ orally. The test was performed 30 minutes after oral administration.

\section{Acknowledgements}

I am thankful for Prof. Dr. Ashraf Bahai Abdul Naim, Professor of Pharmacology, Ain Shams University of performing all biological screening. We are also grateful to Mr. Magdy Ghazy, NMR chemist at King Fahd Medical Research Center, King Abdulaziz University for assisting in 1H NMR spectroscopy.

\section{REFERENCES}

[1] “Cancer/Anticancer Peptides Section, Part VII,” In: A. J. Kasten, Ed., Handbook of Biologically Active Peptides, Academic Press, Waltham, 2006.

http://www.sciencedirect.com/science/book/97801236944 23\#ancSE7.

[2] R. M. Freidenger, "Design and Synthesis of Novel Bioactive Peptides and Peptidomimetics," Journal of Medicinal Chemistry, Vol. 46, No. 26, 2003, pp. 5553-5566. doi:10.1021/jm030484k

[3] P. B. McKay, M. B. Peters, G. Carta, C. T. Flood, E. Dempsey, A. Bell, C. Berry, D. G. Lloyd and D. Fayne, "Identification of Plasmepsin Inhibitors as Selective Antimalarial Agents Using Ligand Based Drug Design," Bioorganic \& Medicinal Chemistry Letters, Vol. 21, No. 11, 2011, pp. 3335-3341. doi:10.1016/j.bmcl.2011.04.015

[4] T. T. H. Nguyen, H. Ryu, S. Lee, S. Hwang, V. Breton, J. H. Rhee and D. Kim, "Virtual Screening Identification of Novel Severe Acute Respiratory Syndrome 3C-Like Protease Inhibitors and in Vitro Confirmation," Bioorganic \& Medicinal Chemistry Letters, Vol. 21, No. 10, 2011, pp. 3088-3091. doi:10.1016/j.bmcl.2011.03.034

[5] Y. K. Peterson, X. S. Wang, P. J. Casey and A. Tropsha, "Discovery of Geranylgeranyltransferase-I Inhibitors with Novel Scaffolds by the Means of Quantitative StructureActivity Relationship Modeling, Virtual Screening, and Experimental Validation," Journal of Medicinal Chemistry, Vol. 52, No. 14, 2009, pp. 4210-4220. doi:10.1021/jm8013772

[6] R. B. Perni, S. C. Conway, S. K. Ladner, K. Zaifert, M. J. Otto and R. W. King, "Phenylpropenamide Derivatives as Inhibitors of Hepatitis B Virus Replication," Bioorganic \& Medicinal Chemistry Letters, Vol. 10, No. 23, 2000, pp. 2687-2690. doi:10.1016/S0960-894X(00)00544-8

[7] E. M. Al-Arab, B. Ezz, M. Wahid and H. H. Maharram, "Synthesis and Evaluation of Antitumor and Antiviral Screening of Some Newer Aryl Acrylamide Derivatives," Mansoura Journal of Pharmaceutical Sciences, Vol. 16, 2000, pp. 98-113.

[8] F. Shi, A. Dai, X. Zhang, B. Jiang and S. Tu, "Facile Diversity-Oriented Synthesis of Novel Dipeptide Mimetic Compounds Containing Bioactive Molecular Skeletons Under Microwave Irradiation," ACS Combinatorial Science, Vol. 13, No. 2, 2011, pp. 147-153. 


\section{doi:10.1021/co1000458}

[9] J. Shan and J. J. Zheng, "Optimizing Dvl PDZ Domain Inhibitor by Exploring Chemical Space," Journal of Computer-Aided Molecular Design, Vol. 23, No. 1, 2009, pp. 37-47. doi:10.1007/s10822-008-9236-1

[10] J. Peters, "Pharmacological Promiscuity and Molecular Properties," In: J. Peters, Ed., Polypharmacology in Drug Discovery, Wiley, Hoboken, 2012, pp. 47-59. doi:10.1002/9781118098141.ch3

[11] S. C. Khadse, G. S. Talele and S. S. Agrawal, "Aminocarbonyl Arylvinylbenzamides as Gastric Sparing Anti-Inflammatory Agents," Pharmaceutical \& Medicinal Chemistry, Vol. 344, No. 5, 2011 pp. 292-300. doi:10.1002/ardp.201000096

[12] B. H. Banu and K. Bharathi, "Synthesis and Analgesic Activity of Some N-Protected Dehydrophenylalanine Dipeptides," Journal of Pharmacy Research, Vol. 3, No. 10, 2010, pp. 2450-2452.

[13] M. S. Mohamed, R. K. Mahmoud, A. I. Sayed and M. E. El-Araby, "Potent Anticonvulsant 1H-Imidazol-5(4H)-One Derivatives with Low Neurotoxicity," The Open Medicinal Chemistry Journal, Vol. 2, No. 2, 2012, pp. 24-29.

[14] A. Jemal, F. Bray, M. M. Center, J. Ferlay, E. Ward and D. Forman, "Global Cancer Statistics," A Cancer Journal for Clinicians, Vol. 61, No. 2, 2011, pp. 69-90. doi:10.3322/caac. 20107

[15] P. Skehan, R. Storeng, D. Scudiero, A. Monks, J. McMahon, D. Vistica, J. T. Warren, H. Bokesch, S. Kenney and M. R. Boyd, "New Colorimetric Cytotoxicity Assay for Anticancer-Drug Screening," Journal of the National Can- cer Institute, Vol. 82, No. 13, 1990, pp. 1107-1112. doi:10.1093/jnci/82.13.1107

[16] P. Ertl, B. Rohde and P. Selzer, "Fast Calculation of Molecular Polar Surface Area as a Sum of Fragment Based Contributions and Its Application to the Prediction of Drug Transport Properties," Journal of Medicinal Chemistry, Vol. 43, No. 20, 2000, pp. 3714-3717. doi:10.1021/jm000942e

[17] J. J. Luszczki, E. Wojda, M. Andres-Mach, W. Cisowski, M. Glensk, K. Glowniak and S. J. Czuczwar, “Anticonvulsant and Acute Neurotoxic Effects of Imperatorin, Osthole and Valproate in the Maximal Electroshock Seizure and Chimney Tests in Mice: A Comparativestudy," Epilepsy Research, Vol. 85, No. 2, 2009, pp. 293-299.

[18] F. Lombardo, B. W. Dominy and P. J. Feeney, "Experimental and Computational Approaches to Estimate Solubility and Permeability in Drug Discovery and Development Settings," Advanced Drug Delivery Reviews, Vol. 46, No. 1-3, 2001, pp. 3-26. doi:10.1016/S0169-409X(00)00129-0

[19] M. Q. Zhang, "Working with Small Molecules: Rules-ofThumb of 'Drug Likeness'," Methods in Molecular Biology, Vol. 803, 2012, pp. 297-307. doi:10.1007/978-1-61779-364-6 20

[20] N. Saldabols, A. Treilone, L. N. Alekseeva, B. Brizga and K. Medne, "Synthesis and Transformations of Furan Derivatives. VI. Amides of $\beta$-(2-Furyl)- and $\beta$-(5-Nitro-2-furyl)- $\alpha$-acylamidoacrylic Acids and MonoSubstituted 2-Phenyl-4-(5-nitro-2-furfurylidene)-5-imidazolones," Khimiko-Farmatsevticheskii Zhurnal, Vol. 1, 1967, pp. 21-27. 